\title{
The effects of oral antioxidants on the semen of men with idiopathic oligoasthenoteratozoospermia
}

\author{
Ahmed T Alahmar \\ Department of Pharmacology and Toxicology, College of Pharmacy, University of Babylon, Babylon, Iraq
}

It has been estimated that approximately $15 \%$ of reproductive-age couples suffer from infertility. Male factors contribute to almost half of infertility cases, and in many patients the underlying cause of oligoasthenoteratozoospermia is unknown. Accumulating evidence suggests that oxidative stress plays a role as a contributing factor to male infertility, and reactive oxygen species have been shown to impair sperm function and motility and to damage sperm membrane and DNA. Therefore, this review explored the evidence provided by studies published from 2002 to 2017 on the impact of oral antioxidants (vitamin C, vitamin E, L-carnitine, coenzyme Q10, zinc, selenium, and pentoxifylline) on seminal fluid parameters in men with idiopathic oligoasthenoteratozoospermia. Most of the studies were randomized controlled studies that investigated the effect of single or combined antioxidants and reported improvements in at least one semen parameter. The most noteworthy effect that was found was that the use of multiple antioxidants increased sperm motility and concentration. Nonetheless, there is a lack of agreement on the dose, the duration of treatment, and whether individual or combined oral antioxidants should be used. Therefore, the current review provides evidence supporting the use of oral antioxidants in the treatment of infertile men with idiopathic oligoasthenoteratozoospermia.

Keywords: Antioxidants; Idiopathic; Male infertility; Oligoasthenoteratozoospermia; Oxidative stress

\section{Introduction}

Infertility is defined as the inability to achieve successful pregnancy after 12 months of regular unprotected sex. It has been estimated that approximately $15 \%$ of reproductive-age couples suffer from infertility, which has become a global concern [1]. Roughly 70 million couples worldwide are affected by infertility and seek treatment for this condition, and male factors contribute to almost half of cases [2]. Male infertility can be attributed to several conditions, including varicocele, hypogonadism, cryptorchidism, infection, autoimmune diseases, systemic diseases, testicular cancer, and genetic abnormalities. Nevertheless, in around $30 \%-40 \%$ of cases, no known cause is identified, and this condition has been termed as idiopathic oligoasthe-

Received: Mar 2, 2018 · Revised: Mar 29, 2018 · Accepted: Apr 16, 2018

Corresponding author: Ahmed T Alahmar

Department of Pharmacology and Toxicology, College of Pharmacy, University

of Babylon, Hilla, Babylon 51001, Iraq

Tel:+964-7808180900 E-mail:ahmed.t.alahmar@gmail.com

This is an Open Access article distributed under the terms of the Creative Commons Attribution Non-Commercial License (http://creativecommons.org/licenses/by-nc/4.0/) which permits unrestricted non-commercial use, distribution, and reproduction in any medium, provided the original work is properly cited. noteratozoospermia (OAT). Idiopathic OAT includes a combination of low sperm concentration $\left(<15 \times 10^{6} / \mathrm{mL}\right)$, reduced motility (progressive motility $<32 \%$ and total motility $<40 \%)$ and abnormally shaped spermatozoa ( $<30 \%$ normal morphology by the 2010 World Health Organization criteria or $<4 \%$ by the Kruger strict criteria) in men who do not have any disease that could affect their fertility [3].

Oxidative stress has been implicated in the development of many diseases, such as cancer, diabetes, cardiovascular disease, rheumatoid arthritis, liver disease, AIDS (acquired immune deficiency syndrome), Parkinson disease, and motor neuron disease [4]. Reactive oxygen species (ROS) levels have been also linked to male infertility, and studies have reported higher levels of ROS and suppressed antioxidant capacity in the semen of infertile men in comparison to their fertile counterparts [5-7]. ROS are normally present to some extent in seminal plasma as they are required for capacitation, the acrosome reaction, and fertilization, but the excessive production of ROS triggered by inflammatory cells has detrimental effects on sperm [8]. The adverse effects of ROS on sperm encompass reduced sperm motility, DNA fragmentation, impaired hyperactivation and oocyte fusion, damage to the sperm membrane due to lipid peroxidation, and 
poor chromatin packing [9-11]. The DNA damage due to ROS can be in the form of base modifications, attacks on phosphodiester bonds, point mutations, deletions, and frameshift mutations $[12,13]$. The resultant sperm damage can decrease the fertilization rate, reduce implantation, hinder embryonic development, and increase the risk of miscarriage and birth defects [14-18]. In addition, increased oxidative stress can adversely affect the proportions of polyunsaturated fatty acids, which are important for providing fluidity during membrane fusion events such as the acrosome reaction, sperm-egg interactions, and sperm motility [19].

Maintenance of redox homeostasis is governed by a balance between ROS production and antioxidants in the body. Antioxidants can be endogenous or exogenous. Endogenous antioxidants are classified into enzymatic antioxidants, such as catalase, superoxide dismutase, glutathione peroxidase, and non-enzymatic antioxidants such as glutathione, vitamin $E$, vitamin $A$, vitamin $C$, pyruvate, taurine, urate, coenzyme Q10 (CoQ10), and L-carnitine (LC) [20]. Exogenous antioxidants, such as vitamin E, vitamin C, and carotenoids, are mainly obtained from dietary sources [21]. In addition, semen also contains a recently recognized family of antioxidant enzymes known as peroxidoxins [12].

There is accumulating evidence that supports the use of oral antioxidants for the treatment of men with idiopathic OAT. The rationale is that seminal oxidative stress is a potential contributing factor to infertility due to decreased levels of seminal antioxidants [18]. Oral antioxidant therapy helps to scavenge seminal ROS and restore the redox balance [18]. The various available oral antioxidants encompass vitamin A; vitamin C; vitamin E; LC; CoQ10; N-acetyl cysteine; micronutrients such as zinc, selenium, and pentoxifylline (PTX); and combinations of these oral antioxidants. Therefore, this review aimed to explore the evidence provided by studies published from 2002 to 2017 on the impact of oral antioxidants (vitamin C, vitamin E, LC, CoQ10, zinc, selenium, and PTX) on seminal fluid parameters in men with idiopathic OAT.

\section{Vitamin C}

Vitamin C, also known as ascorbic acid, is a water-soluble vitamin freely available in citrus fruits and fresh berries that is known to have many beneficial effects through its antioxidant properties. Various studies have been carried out on the effects of ascorbic acid supplementation on sperm function. A prospective study conducted by Akmal et al. [22] observed that vitamin C supplementation ( $2 \mathrm{~g} /$ day) in infertile men with idiopathic oligozoospermia induced a significant increase in sperm motility and sperm count, as well as an increase in the percentage of normal spermatozoa, but there was no placebo group in that study. Eslamian et al. [23] performed a case-control study to assess associations between dietary patterns and asthenozoospermia in 107 patients and 235 age-matched controls. It was observed that a diet rich in vitamin C, vitamin E, vitamin D, zinc, folate, total fiber, polyunsaturated fatty acids, and selenium was significantly associated with a lower risk of asthenozoospermia. Vitamin C supplementation (250 mg twice daily) improved sperm motility and normal morphology, but not sperm count, in 115 infertile men following varicocele surgery in a double-blind randomized controlled trial [24]. Kobori et al. [25] investigated the effect of administration of vitamin C (80 mg), vitamin E (40 mg), and CoQ10 (120 mg) in 169 men with oligoasthenozoospermia and observed improvements in sperm concentration and motility after 3 and 6 months of treatment, as well as a pregnancy rate of $28.4 \%$ (seven after 3 months, eight after 6 months of treatment). A study of male rats demonstrated that ascorbic acid could reverse cyclophosphamide-induced testicular oxidative stress and testicular androgenic disorders [26]. Another study explored the effects of astaxanthin (100 mg/kg), vitamin E (100 mg/kg), vitamin C $(100 \mathrm{mg} / \mathrm{kg})$, and calorie restriction in male rats for 86 days and reported that antioxidant supplementation with or without calorie restriction did not have a significant influence on isoprostane, which is an oxidative stress marker. The antioxidant combination partially improved male infertility [27]. Another study reported that vitamin C mitigated the cyclophosphamide-induced reduction in seminal fluid parameters in rats attributed to oxidative stress [28].

\section{Vitamin E}

Vitamin E, also known as alpha-tocopherol, is a fat-soluble antioxidant vitamin found in almonds, avocados, spinach, and sweet potatoes that neutralizes free radicals and protects the cellular membrane against damage from ROS. It prevents lipid peroxidation and enhances the function of other antioxidants. The recommended daily allowance of vitamin $\mathrm{E}$ is $12 \mathrm{mg}$ according to the European Commission Directive 2008/100/EC [29]. An interventional, placebo-controlled study of infertile men by Greco et al. [30] showed that 2-month supplementation of vitamin E (1 g/day) and vitamin C (1 g/day) resulted in a significant reduction of sperm DNA damage. However, there was no significant association between vitamin $C$ and $E$ intake and sperm parameters such as motility and concentration. In a prospective study of 690 men suffering from idiopathic OAT, selenium supplements $(200 \mu \mathrm{g})$ in combination with vitamin $\mathrm{E}$ (400 IU) were administered for a period of 100 days, and in $52.6 \%$ of cases, there was an overall improvement in sperm motility, morphology, or both. Moreover, spontaneous pregnancy occurred in $10.8 \%$ of cases in the treatment group [31]. Another study by Greco and colleagues [32] showed that treatment with $1 \mathrm{~g}$ of vitamin $\mathrm{E}$ and vitamin $\mathrm{C}$ resulted in improved success rates of intracytoplasmic sperm injection (ICSI) 
and decreased sperm DNA damage.

In a randomized controlled trial conducted by ElSheikh et al. [33], 90 men with idiopathic OAT were divided into three groups that received vitamin $\mathrm{E}$ ( $400 \mathrm{mg} /$ day), clomiphene citrate ( $25 \mathrm{mg} /$ day), or a combination of vitamin $\mathrm{E}$ and clomiphene citrate for 6 months. Semen examinations post-therapy revealed that the sperm concentration and motility improved in all three groups, but to a greater extent in the combination group. It was found that $\mathrm{CoQ} 10$ and vitamin E plasma concentrations were reduced in 40 infertile men diagnosed with OAT. Accordingly, CoQ10 and vitamin E can be used as potential metabolic biomarkers for the diagnosis and treatment of male infertility [34].

\section{L-carnitine}

LC, or 3-aminobutyric acid, is a naturally occurring substance found in red meat and dairy products that is required for human metabolism. This molecule is involved as an intermediate in bioenergetic processes, where it plays an important role in the formation of acyl carnitine esters of long-chain fatty acids [35]. The concentration of LC is 2,000-fold greater in the epididymis than in the blood plasma, which is due to the active secretory process in the epididymis [35,36]. In a randomized controlled trial performed by Moslemi Mehni et al. [37] on 212 infertile men with idiopathic OAT, the combination of LC (500 mg) and PTX (400 mg) twice daily for a 3-month duration resulted in increases in all sperm parameters and improved outcomes of assisted reproductive technologies. An observational study of infertile men in Pakistan showed that seminal free LC levels were lower in infertile men than in fertile controls. Furthermore, strong positive correlations were found between seminal LC levels and sperm count, motility, and normal morphology [38]. Another study showed that seminal free LC levels were lower in infertile men than in fertile controls, and the lowest concentration was observed in the azoospermic group [39].

Lenzi et al. [40] carried out a double-blind placebo-controlled trial to assess the effect of LC supplements in 56 men with idiopathic OAT. The intervention group received $2 \mathrm{~g} /$ day of LC and $1 \mathrm{~g} /$ day of L-acetyl carnitine (LAC) for a duration of 6 months. Significant relationships between LC/LAC and improvements in all semen parameters were reported, and this trend was more prominent in infertile men with lower sperm motility at baseline. Another double-blind trial was conducted by Garolla et al. [41], examining the effects of LC therapy on phospholipid hydroperoxide glutathione peroxidase (PHGPX) levels in men suffering from idiopathic OAT. PHGPX levels were assessed at baseline and after receiving LC ( $2 \mathrm{~g} /$ day) for a period of 3 months. Semen analysis showed that LC therapy significantly improved sperm motility, and that the PHGPX levels were restored to normal. Balercia et al. [42] also carried out a randomized, placebo-controlled, double- blind trial of 59 men with idiopathic OAT, and assessed total oxygen radical scavenging capacity as well as sperm motion kinetics. A combination therapy utilizing LC and LAC ( $3 \mathrm{~g} /$ day) or placebo for a period of 6 months significantly increased sperm motility and total oxygen radical scavenging capacity. Nine pregnancies were achieved during this period, and five of them were in couples where the male partner was receiving combination therapy. Another study randomized 135 patients with asthenozoospermia to receive either LC ( $2 \mathrm{~g} /$ day) with vitamin $\mathrm{E}$ or vitamin $\mathrm{E}$ only for 3 months. The first group showed an increase in sperm motility, but no improvements were found in sperm density or normal morphology [43].

\section{Coenzyme Q10}

CoQ10 is a nonenzymatic antioxidant that is responsible for the protection of cells against lipid peroxidation-induced damage. It is found in organic meats, beef, soy oil, sardines, and peanuts. Lipid peroxidation is a significant feature of ROS-mediated cellular damage, in which cellular membrane fluidity is altered, membrane potential is reduced, harmful lipid epoxides are generated. CoQ10 protects lipids by scavenging the superoxide anion and peroxides, and it also helps in the mitochondrial electron transport chain, which generates adenosine triphosphate [44-46]. In a study of male Wistar rats with high low-density lipoprotein and oxidized low-density lipoprotein levels, protective effects of CoQ10 and LC were observed. It has been hypothesized that hypercholesterolemia can lead to fertility issues, and the protective effects of LC and CoQ10 supplementation led to significant improvements in sperm parameters, sperm function, and reproductive hormone profiles [4].

A randomized placebo-controlled trial carried out by Safarinejad [47] showed that CoQ10 therapy (300 mg for a 26-week period) in 212 infertile men suffering from idiopathic OAT resulted in a significant improvement in sperm count, motility, and morphology. Another double-blind placebo-controlled clinical trial investigated the effects of the reduced form of $\mathrm{CoQ} 10$ on sperm parameters and seminal plasma antioxidant capacity in 228 men with idiopathic OAT. The patients received oral supplementation of CoQ10 (200 mg) for a period of 26 weeks, and they showed improvements in sperm density, motility, and morphology. The study demonstrated that during the off-drug period, the sperm parameters gradually returned to their baseline values, but the observed differences were still significant [48]. In an open-label prospective study by Safarinejad [49], in 287 infertile men with idiopathic OAT, treatment with CoQ10 (300 mg orally, twice daily) for 12 months resulted in improvements in sperm concentration, progressive motility, and normal sperm morphology, and a pregnancy rate of $34.1 \%$ was reported. In contrast, in a clinical trial, Nadjarzadeh et al. [50] observed that supplementation with 
CoQ10 (200 mg for a 16-week period) produced no significant changes in sperm parameters, but significantly increased the seminal total antioxidant capacity. In another clinical trial performed by Nadjarzadeh and colleagues [51], the effects of CoQ10 supplementation on seminal antioxidants and oxidative stress apart from sperm parameters in men with idiopathic OAT were studied. It was observed that CoQ10 supplementation for a period of 3 months improved the activity of enzymatic antioxidants, such as catalase and superoxide dismutase. It was also shown that there were lower 8-isoprostane (oxidative stress marker) levels in the intervention group that received CoQ10 therapy. The researchers concluded that CoQ10 levels were significantly correlated with key semen parameters such as sperm morphology, motility, and density due to improvements in the total antioxidant capacity. Thakur et al. [52] showed that supplementation with $150 \mathrm{mg}$ of CoQ10 significantly enhanced sperm parameters in infertile men. Finally, a meta-analysis of the effect of CoQ10 supplementation in infertile men showed that global improvements in sperm parameters such as motility, morphology, and concentration accompanied increased concentrations of $\mathrm{CoQ} 10$ in semen. These results, however, must be interpreted with caution because they do not necessarily indicate an increased chance of pregnancy or live birth [53].

\section{Zinc}

Zinc deficiency has been postulated as a putative contributing factor to male factor infertility [54]. Zinc is the second most abundant trace element found in human tissue, following iron. Food sources of zinc include red and white meat, fish, and milk, and the World Health Organization estimated that about one-third of the global population is deficient in zinc [55]. The trace element zinc serves as a cofactor for numerous enzymes responsible for cellular development, such as DNA transcription and protein synthesis. Zinc plays a pivotal role in testicular development, steroidogenesis, the synthesis and secretion of luteinizing hormone and follicle-stimulating hormone, gonadal differentiation, the formation and maturation of spermatozoa, the acrosome reaction, and fertilization [56,57]. A recent systematic review concluded that seminal zinc levels were lower in infertile men and that zinc supplementation increased semen volume, sperm motility, and normal sperm morphology [5]. Another study of 150 infertile men demonstrated positive correlations between seminal plasma zinc levels and semen parameters and serum free testosterone [58]. In a clinical trial conducted in Iraq, semen samples were acquired from 60 asthenozoospermic infertile men and 60 agematched fertile men. Zinc sulfate supplementation, with a daily dose of $440 \mathrm{mg}$, was given to the infertile men for a period of 3 months, and the qualitative and quantitative characteristics of semen, along with peroxynitrite, arginase, and nitric oxide (NO) synthase activity, were assessed. The peroxynitrite levels and NO synthase activity were significantly higher in the infertile men than in the fertile group whereas arginase activity was higher in the fertile group than in the infertile men. Moreover, peroxynitrite, NO synthase, and arginase levels were restored to normal values following zinc supplementation in infertile men. Semen volume, sperm count, and progressive sperm motility significantly improved following zinc supplementation in asthenozoospermic men [59]. In another study, the same researchers investigated the effect of zinc supplementation on zinc binding protein levels and qualitative and quantitative semen characteristics. Semen samples were collected from 37 fertile men and 37 subfertile men with asthenozoospermia who were given zinc supplementation (440 mg/day) for a 3-month period. The results of the study showed that zinc supplementation significantly increased semen volume, sperm motility, and sperm count in the subfertile men [60]. A combination of zinc and folic acid supplementation was studied in men suffering from OAT. In this randomized, double-blinded, placebo-controlled trial, 83 men with OAT received zinc $(220 \mathrm{mg} /$ day) and folic acid ( $5 \mathrm{mg} /$ day) orally for a period of 16 weeks. The study did not show improvements in sperm quality in subfertile men suffering from OAT, even after adjusting for the placebo effect [61].

\section{Selenium}

Selenium is an essential trace element that plays an important role in sperm formation and testosterone synthesis [62]. At least 25 selenoproteins have been identified in humans and animals, and these selenoproteins help to maintain the structural integrity of sperm [37]. A placebo-controlled clinical trial was performed on men with idiopathic OAT who received $200 \mu \mathrm{g}$ of selenium orally along with 600 $\mathrm{mg}$ of $\mathrm{N}$-acetyl-cysteine or a similar regimen of placebo for a period of 26 weeks, followed by a 30-week treatment-free period. Significant positive correlations were found between seminal plasma concentrations of selenium and $\mathrm{N}$-acetyl cysteine and mean sperm concentration, motility, and normal morphology [63]. Another clinical trial was conducted on 54 infertile men and healthy controls who received supplementation with vitamin $\mathrm{E}(400 \mathrm{mg})$ and selenium (225 $\mu \mathrm{g}$ ) for a 3-month period, while a placebo group received vitamin $B$ (4.5 g/day) for the same duration. In comparison to vitamin B, selenium and vitamin $E$ supplementation resulted in a significant decrease in an oxidative stress marker (malondialdehyde), and sperm motility and viability were inversely correlated with malondialdehyde levels [64]. Selenium-fortified probiotics reduced triglyceride levels and improved sperm count, mobility, and morphology in obese rats [65]. 


\section{Pentoxifylline}

PTX is a derivative of xanthine that increases local blood flow by increasing the deformability of red blood cells and decreasing blood viscosity [66]. PTX has been recommended as an artificial sperm movement enhancer, and it has been shown to be particularly useful in patients with asthenozoospermia, who exhibit decreased sperm motility in their ejaculate [67]. An in vitro study showed that PTX significantly improved sperm movement in asthenozoospermic semen samples without adverse effects on sperm DNA or chromatin integrity during a vitrification program [68]. It was also shown that PTX had a positive effect on ICSI outcomes, including fertilization, embryo quality, and pregnancy rates, in asthenozoospermic patients [67]. Safarinejad [69] performed a randomized controlled trial on men with idiopathic OAT, investigating the response of semen parameters to supplementation with PTX (400 mg twice daily) for a 24-week treatment phase followed by a 12-week treatment-free period. The results of that study showed a significant improvement in seminal parameters such as concentration, motility, and morphology. An increase was also observed in the acrosome reaction in the PTX group. Another study observed that PTX (1,200 mg) in combination with $5 \mathrm{mg}$ of folic acid and $66 \mathrm{mg}$ of zinc sulfate administered orally every day for a period of 12 weeks in patients suffering from varicocele improved sperm morphology starting in the 4th week of treatment [70].

\section{Combination of oral antioxidants}

The current trend is to employ multiple antioxidants to treat male infertility in order to achieve synergistic antioxidant effects. Gharagozloo et al. [13] found that a combination of LC (500 mg), folic acid $(450 \mu \mathrm{g})$, vitamin C (60 mg), lycopene (10 mg), selenium (55 $\mu \mathrm{g})$, vita$\min \mathrm{E}(200 \mathrm{mg})$, and zinc (10 mg) significantly reduced levels of 8-hydroxydeoxyguanosine, a marker of DNA damage, in sperm cells in mice. They also observed that in a scrotal heat stress model, pretreatment with antioxidants led to $74 \%$ of female mice becoming pregnant, resulting in 427 fetuses, which constituted an improvement in fertility. The effects of another combination of vitamin C (100 mg), vitamin $E(100 \mathrm{mg})$, folic acid $(500 \mu \mathrm{g})$, zinc $(25 \mathrm{mg})$, selenium (100 $\mu \mathrm{g})$, $\mathrm{N}$-acetyl cysteine (50 mg), LC (300 mg), citrulline (300 mg), lycopene (4 mg), and CoQ10 (15 mg) on sperm quality were investigated in 147 patients who underwent in vitro fertilization (IVF). The patients were categorized as OAT and non-OAT men, and following the first and second sperm analyses, they received vitamin C (100 mg), vitamin E (100 mg), folic acid (500 $\mu \mathrm{g})$, zinc (25 mg), selenium (100 $\mu \mathrm{g})$, $\mathrm{N}$-acetyl-L-cysteine (50 mg), and LC (300 mg). Significant improvements were found in sperm concentration, motility, and normal morphology following micronutrient and vitamin supplementation, and the effects were stronger in men with OAT with restricted sperm parameters. The sperm parameters that improved were motility, sperm count, and nuclear vacuolization [71]. These findings are congruent with those of our recent study that demonstrated that daily supplementation with vitamin C ( $90 \mathrm{mg} /$ day), vitamin $\mathrm{E}$ ( $15 \mathrm{mg} /$ day), CoQ10 (4 mg/day), selenium (30 $\mu \mathrm{g} /$ day), and zinc ( $5 \mathrm{mg} /$ day) for 3 months improved sperm concentration, progressive motility, and total motility in men with idiopathic oligoasthenozoospermia [72].

Abad et al. [73] performed a study to assess the effect of oral antioxidant supplementation on the sperm dynamics of DNA fragmentation in a cohort of 20 infertile men with asthenoteratozoospermia. $\mathrm{Pa}$ tients received 1,500 $\mathrm{mg}$ of $\mathrm{LC}, 60 \mathrm{mg}$ of vitamin C, $20 \mathrm{mg}$ of CoQ10, $10 \mathrm{mg}$ of vitamin $\mathrm{E}, 10 \mathrm{mg}$ of zinc, $200 \mu \mathrm{g}$ of folic acid, $50 \mu \mathrm{g}$ of selenium, and $1 \mu \mathrm{g}$ of vitamin B12 for a duration of 3 months. Semen analysis showed that the proportion of degraded sperm significantly decreased, and significant improvements were found in sperm concentration, motility, and morphology. There was also a significant improvement in DNA integrity, and It was concluded that treatment with multiple antioxidants can open a significant therapeutic window in the treatment of male infertility. Piomboni et al. [74] observed that a combination of antioxidants including beta-glucan (20 mg), fermented papaya (50 mg), lactoferrin ( $97 \mathrm{mg}$ ), vitamin C (30 mg), and vitamin $\mathrm{E}$ (5 mg), administered twice daily for a period of 3 months, yielded significant improvements in the percentage of morphologically normal sperm cells and progressive sperm motility, as well as a decrease in leukocyte concentrations in asthenoteratozoospermic patients.

Gopinath et al. [75] conducted a placebo-controlled trial in men suffering from OAT and initiated treatment with the oral administration of a fixed-dose combination (FDC) of multiple antioxidants (50 $\mathrm{mg}$ of CoQ10, $500 \mathrm{mg}$ of LC, $2.5 \mathrm{mg}$ of lycopene, and $12.5 \mathrm{mg}$ of zinc). The participants were allocated into three groups, which received two tablets twice daily of FDC, one tablet of FDC and one tablet of placebo, or two tablets of placebo twice daily for 180 days. It was found that FDC administration was safe and effective at improving sperm count and sperm motility in the first interval (90 days), and further improvement was observed in the second interval, at 180 days. Tremellen et al. [76] performed a prospective, randomized, double-blind, placebo-controlled trial in 60 couples with male infertility. The subjects were randomly assigned to one capsule per day containing $400 \mathrm{lU}$ of vitamin E, $50 \mathrm{mg}$ of vitamin C, $6 \mathrm{mg}$ of lycopene, $25 \mathrm{mg}$ of zinc, $25 \mu \mathrm{g}$ of selenium, $5 \mathrm{mg}$ of folic acid, and 1,000 mg of garlic or placebo for 3 months prior to their partner's IVF or ICSI cycle. The group that received antioxidant supplementation showed a statistically significant improvement in the viable pregnancy rate (38.5\%) in comparison to the control group (16\%). There were no significant changes in the oocyte fertilization rate or embryo quality between the control group and the group that received antioxidants. 
Another study showed that a combination of LC (145 mg), acetylLC (64 mg), fructose (250 mg), citric acid (50 mg), selenium (50 $\mu \mathrm{g})$, CoQ10 (20 mg), zinc (10 mg), ascorbic acid (90 mg), cyanocobalamin $(15 \mu \mathrm{g})$, and folic acid $(200 \mu \mathrm{g})$ administered once daily for a duration of 4 months in 96 men with idiopathic asthenoteratozoospermia resulted in a significant increase in progressive sperm motility, and 16 patients achieved pregnancy during the course of the trial [77]. In a study conducted by Ghanem et al. [78], it was found that in men suffering from idiopathic OAT, supplementation with a combination of antioxidants including vitamin E (400 mg/day) and clomiphene citrate ( $25 \mathrm{mg} /$ day) resulted in a significant increase in sperm concentration and progressive sperm motility. The rate of spontaneous pregnancy was higher (36.7\%) in the treatment group than in the placebo group (13.3\%). Comhaire et al. [79] showed that the combination of $\mathrm{N}$-acetyl cysteine or vitamins $\mathrm{A}$ or $\mathrm{E}$ and essential fatty acid supplementation in infertile men was associated with a significant reduction in ROS activity and an increase in the acrosome reaction, but nonsignificant improvement was shown in sperm motility and morphology, with only a slight increase in the sperm concentration in oligozoospermic men.

\section{Discussion}

Accumulating evidence suggests that semen quality has considerably declined over the past 20 years. This change in semen quality has been linked to environmental factors, metal toxicity, chemicals, radiation, and heat. Furthermore, factors such as obesity, inflammation, pollutants, cigarette smoking, and ROS are negatively correlated with spermatogenesis and sperm DNA integrity [80]. Elevated ROS levels can result in damage to the proteins, lipid membranes, or DNA integrity of spermatozoa, meaning that sufficient antioxidant release is essential for maintaining normal sperm quality and quantity, which ensure fertility in men [18]. Oxidative stress has emerged as a key player in the pathogenesis of male infertility, which can be attributed to an increase in free radicals and ROS that results in cellular and tissue damage $[21,81]$. Antioxidants neutralize oxidative stress, and the seminal plasma contains these substances in order to maintain the redox balance. It has been shown that infertile men have high oxidative stress levels in their seminal plasma, and their antioxidant capacity has been found to be insufficient to combat the corresponding damage $[9,82]$.

The current review provides evidence for the use of oral antioxidants in the treatment of male infertility and their effects on seminal parameters. Several studies have explored the actions of vitamin C, vitamin E, LC, CoQ10, zinc, selenium, and PTX, as monotherapy or polytherapy. Most of these studies were randomized placebo-controlled trials or case-control studies. Various doses were used for each antioxidant, and the duration of treatment ranged between 3 and 6 months. Some studies showed positive effects of these antioxidants on sperm concentration, motility, normal morphology, DNA fragmentation, seminal plasma antioxidant capacity, pregnancy rate, and IVF/ICSI outcomes. The most noteworthy effects, however, were on sperm motility and concentration, especially with the use of multiple oral antioxidants. These positive effects can primarily be attributed to the antioxidant properties of the investigated vitamins and micronutrients and the corresponding reduction of ROS and/or increase in seminal plasma antioxidant capacity $[13,20,83]$. The enhanced effects associated with combinations of antioxidants could be due to their synergistic antioxidant action. Alternatively, the mechanism by which antioxidants increase sperm concentration could be through the suppression of ROS-induced sperm damage or another unidentified mechanism. A small number of other studies, in contrast, demonstrated a lack of impact of these antioxidants on one or more semen parameter. Comparing the outcomes of various studies is challenging for several reasons. While many studies were randomized placebo-controlled trials, others were either open, uncontrolled, prospective studies or case-control studies. The patients included in the studies were also heterogeneous, as some studies included patients with idiopathic OAT and others included patients with idiopathic oligoasthenozoospermia or asthenoteratozoospermia. Furthermore, variability was present in the inclusion and exclusion criteria used to define idiopathic infertility. Further, some trials included a small number of patients, while others had a large number of patients, so the negative results obtained in some studies may be attributed to a small sample size and insufficient power. Finally, there were significant differences in the doses of antioxidants used and the duration of the treatment period; these factors are likely to have affected the improvements in seminal fluid parameters in men with idiopathic OAT.

In the normal physiological state, the seminal plasma contains endogenous antioxidant enzymes that can quench ROS and protect the spermatozoa from damage. However, exogenous antioxidants such as vitamin C, vitamin E, CoQ10, and glutathione have been shown to protect against oxidative stress in men experiencing infertility [84]. A synergy exists between the exogenous and endogenous antioxidants that neutralize the free radicals and ROS in the seminal plasma [85]. The interaction of endogenous and exogenous antioxidants results in redox homeostasis $[85,86]$. Both the synthetic and natural forms of antioxidants have recently been the focus of attention in the field of reproduction and fertility management. Three months of administration of vitamins $A, E$, and $C$ and selenium significantly increased sperm motility [87]. However, in contrast, some studies have reported that treatment with oral antioxidants did not result in a significant improvement in sperm parameters $[79,88]$. Once the positive effects of antioxidant supplementation are con- 
firmed, it remains necessary to optimize the dose and duration of treatment and to identify which seminal parameters benefit the most from certain antioxidants, either as single agents or in combination. In patients experiencing high levels of oxidative stress, doses should be taken for a minimum of 3 months, as the maturation of sperm takes around 72 days [89].

\section{Conclusion}

Most of the studies in this review were randomized controlled studies that explored the effects of oral antioxidants in men with idiopathic OAT and reported improvements in at least one semen parameter (motility, concentration, normal morphology, and antioxidant capacity), but the most noteworthy effect was that the use of multiple antioxidants for 3-6 months increased sperm motility and concentration. There is, however, lack of agreement on the dose and duration of treatment and whether individual or combined oral antioxidants should be used. Future directions include identifying the underlying molecular mechanisms that explain the specific effects of some antioxidants on semen parameters, optimizing the dose and duration of therapy and the choice between individual or combined therapy, and measuring ROS and oxidants/antioxidants in seminal plasma. The current review provides cumulative evidence of the positive role of oral antioxidants in the treatment of infertile men with idiopathic OAT. Further large-scale randomized placebo-controlled trials are required to consolidate the evidence provided in the current review.

\section{Conflict of interest}

No potential conflict of interest relevant to this article was reported.

\section{Acknowledgments}

The author would like to thank Dr. Hayder O Hashim, Dr. Asseel K Shaker, and Dr. Raghdan Z Al-Saad at the College of Pharmacy, University of Babylon, Iraq for reviewing this work and providing valuable comments and suggestions for improving this paper prior to submission.

\section{References}

1. Sharlip ID, Jarow JP, Belker AM, Lipshultz LI, Sigman M, Thomas AJ, et al. Best practice policies for male infertility. Fertil Steril 2002;77:873-82.

2. Boivin J, Bunting L, Collins JA, Nygren KG. International estimates of infertility prevalence and treatment-seeking: potential need and demand for infertility medical care. Hum Reprod 2007;
22:1506-12.

3. Cooper TG, Noonan E, von Eckardstein S, Auger J, Baker HW, Behre HM, et al. World Health Organization reference values for human semen characteristics. Hum Reprod Update 2010;16: 231-45.

4. Ghanbarzadeh S, Garjani A, Ziaee M, Khorrami A. CoQ10 and Lcarnitine attenuate the effect of high $L D L$ and oxidized $L D L$ on spermatogenesis in male rats. Drug Res (Stuttg) 2014;64:510-5.

5. Zhao J, Dong X, Hu X, Long Z, Wang L, Liu Q, et al. Zinc levels in seminal plasma and their correlation with male infertility: a systematic review and meta-analysis. Sci Rep 2016;6:22386.

6. Dorostghoal M, Kazeminejad SR, Shahbazian N, Pourmehdi M, Jabbari A. Oxidative stress status and sperm DNA fragmentation in fertile and infertile men. Andrologia 2017;49:e12762.

7. Agarwal A, Virk G, Ong C, du Plessis SS. Effect of oxidative stress on male reproduction. World J Mens Health 2014;32:1-17.

8. Oluwakemi 0 , Olufeyisipe A. DNA fragmentation and oxidative stress compromise sperm motility and survival in late pregnancy exposure to omega-9 fatty acid in rats. Iran J Basic Med Sci 2016; 19:511-20.

9. Agarwal A, Roychoudhury S, Bjugstad KB, Cho CL. Oxidation-reduction potential of semen: what is its role in the treatment of male infertility? Ther Adv Urol 2016;8:302-18.

10. Aitken RJ, Krausz C. Oxidative stress, DNA damage and the $Y$ chromosome. Reproduction 2001;122:497-506.

11. Cocuzza M, Sikka SC, Athayde KS, Agarwal A. Clinical relevance of oxidative stress and sperm chromatin damage in male infertility: an evidence based analysis. Int Braz J Urol 2007;33:603-21.

12. Agarwal A, Saleh RA, Bedaiwy MA. Role of reactive oxygen species in the pathophysiology of human reproduction. Fertil Steril 2003;79:829-43.

13. Gharagozloo P, Gutierrez-Adan A, Champroux A, Noblanc A, Kocer $A$, Calle $A$, et al. A novel antioxidant formulation designed to treat male infertility associated with oxidative stress: promising preclinical evidence from animal models. Hum Reprod 2016;31: 252-62.

14. Henkel R, Kierspel E, Hajimohammad M, Stalf T, Hoogendijk C, Mehnert $\mathrm{C}$, et al. DNA fragmentation of spermatozoa and assisted reproduction technology. Reprod Biomed Online 2003;7:47784.

15. Ozmen B, Koutlaki N, Youssry M, Diedrich K, Al-Hasani S. DNA damage of human spermatozoa in assisted reproduction: origins, diagnosis, impacts and safety. Reprod Biomed Online 2007;14:384-95.

16. Lewis SE, Simon L. Clinical implications of sperm DNA damage. Hum Fertil (Camb) 2010;13:201-7.

17. Tarozzi N, Bizzaro D, Flamigni C, Borini A. Clinical relevance of 
sperm DNA damage in assisted reproduction. Reprod Biomed Online 2007;14:746-57.

18. Zini A, San Gabriel M, Baazeem A. Antioxidants and sperm DNA damage: a clinical perspective. J Assist Reprod Genet 2009;26: 427-32.

19. Zini A, Al-Hathal N. Antioxidant therapy in male infertility: fact or fiction? Asian J Androl 2011;13:374-81.

20. Adewoyin M, Ibrahim M, Roszaman R, Isa ML, Alewi NA, Rafa AA, et al. Male infertility: the effect of natural antioxidants and phytocompounds on seminal oxidative stress. Diseases 2017;5:E9.

21. Imamovic Kumalic S, Pinter B. Review of clinical trials on effects of oral antioxidants on basic semen and other parameters in idiopathic oligoasthenoteratozoospermia. Biomed Res Int 2014; 2014:426951.

22. Akmal M, Qadri JQ, AI-Waili NS, Thangal S, Haq A, Saloom KY. Improvement in human semen quality after oral supplementation of vitamin C. J Med Food 2006;9:440-2.

23. Eslamian G, Amirjannati N, Rashidkhani B, Sadeghi MR, Hekmatdoost A. Nutrient patterns and asthenozoospermia: a case-control study. Andrologia 2017;49:e12624.

24. Cyrus A, Kabir A, Goodarzi D, Moghimi M. The effect of adjuvant vitamin $C$ after varicocele surgery on sperm quality and quantity in infertile men: a double blind placebo controlled clinical trial. Int Braz J Urol 2015;41:230-8.

25. Kobori Y, Ota S, Sato R, Yagi H, Soh S, Arai G, et al. Antioxidant cosupplementation therapy with vitamin $C$, vitamin $E$, and coenzyme Q10 in patients with oligoasthenozoospermia. Arch Ital Urol Androl 2014;86:1-4.

26. Das UB, Mallick M, Debnath JM, Ghosh D. Protective effect of ascorbic acid on cyclophosphamide- induced testicular gametogenic and androgenic disorders in male rats. Asian J Androl 2002;4:201-7.

27. Vahidinia A, Rahbar AR, Shakoori Mahmoodabadi MM. Effect of astaxanthin, vitamin $\mathrm{E}$, and vitamin $\mathrm{C}$ in combination with calorie restriction on sperm quality and quantity in male rats. J Diet Suppl 2017;14:252-63.

28. Shabanian S, Farahbod F, Rafieian M, Ganji F, Adib A. The effects of vitamin $C$ on sperm quality parameters in laboratory rats following long-term exposure to cyclophosphamide. J Adv Pharm Technol Res 2017;8:73-9.

29. European Commission. Commission directive 2008/100/EC of 28 October 2008 amending council directive 90/496/EEC on nutrition labelling for foodstuffs as regards recommended daily allowance, energy conversion factors and definitions. Official J Eur Union 2008;L285/9.

30. Greco E, lacobelli M, Rienzi L, Ubaldi F, Ferrero S, Tesarik J. Reduction of the incidence of sperm DNA fragmentation by oral anti- oxidant treatment. J Androl 2005;26:349-53.

31. Moslemi MK, Tavanbakhsh S. Selenium-vitamin E supplementation in infertile men: effects on semen parameters and pregnancy rate. Int J Gen Med 2011;4:99-104.

32. Greco E, Romano S, lacobelli M, Ferrero S, Baroni E, Minasi MG, et al. ICSI in cases of sperm DNA damage: beneficial effect of oral antioxidant treatment. Hum Reprod 2005;20:2590-4.

33. ElSheikh MG, Hosny MB, Elshenoufy A, Elghamrawi H, Fayad A, Abdelrahman S. Combination of vitamin $\mathrm{E}$ and clomiphene citrate in treating patients with idiopathic oligoasthenozoospermia: a prospective, randomized trial. Andrology 2015;3:864-7.

34. Gvozdjakova A, Kucharska J, Dubravicky J, Mojto V, Singh RB. Coenzyme Q10, a-tocopherol, and oxidative stress could be important metabolic biomarkers of male infertility. Dis Markers 2015; 2015:827941.

35. Arduini A, Bonomini M, Savica V, Amato A, Zammit V. Carnitine in metabolic disease: potential for pharmacological intervention. Pharmacol Ther 2008;120:149-56.

36. Enomoto A, Wempe MF, Tsuchida H, Shin HJ, Cha SH, Anzai N, et al. Molecular identification of a novel carnitine transporter specific to human testis: insights into the mechanism of carnitine recognition. J Biol Chem 2002;277:36262-71.

37. Moslemi Mehni N, Ketabchi AA, Hosseini E. Combination effect of Pentoxifylline and L-carnitine on idiopathic oligoasthenoteratozoospermia. Iran J Reprod Med 2014;12:817-24.

38. Haseen Ahmed SD, Ahsan S, lqbal T, Ahmed Burney Sl. Relationship of seminal free L-Carnitine with functional spermatozoal characteristics: results from an observational study conducted in a tertiary care hospital of Karachi, Pakistan. J Pak Med Assoc 2017;67:280-4.

39. Ahmed SD, Karira KA, Jagdesh, Ahsan S. Role of L-carnitine in male infertility. J Pak Med Assoc 2011;61:732-6.

40. Lenzi A, Sgro P, Salacone P, Paoli D, Gilio B, Lombardo F, et al. A placebo-controlled double-blind randomized trial of the use of combined I-carnitine and I-acetyl-carnitine treatment in men with asthenozoospermia. Fertil Steril 2004;81:1578-84.

41. Garolla A, Maiorino M, Roverato A, Roveri A, Ursini F, Foresta C. Oral carnitine supplementation increases sperm motility in asthenozoospermic men with normal sperm phospholipid hydroperoxide glutathione peroxidase levels. Fertil Steril 2005;83:35561.

42. Balercia G, Regoli F, Armeni T, Koverech A, Mantero F, Boscaro M. Placebo-controlled double-blind randomized trial on the use of L-carnitine, L-acetylcarnitine, or combined L-carnitine and L-acetylcarnitine in men with idiopathic asthenozoospermia. Fertil Steril 2005;84:662-71.

43. Wang YX, Yang SW, Qu CB, Huo HX, Li W, Li JD, et al. L-carnitine: 
safe and effective for asthenozoospermia. Zhonghua Nan Ke Xue 2010;16:420-2.

44. Ahmadvand H, Tavafi M, Khosrowbeygi A. Amelioration of altered antioxidant enzymes activity and glomerulosclerosis by coenzyme Q10 in alloxan-induced diabetic rats. J Diabetes Complications 2012;26:476-82.

45. Lee BJ, Huang YC, Chen SJ, Lin PT. Coenzyme Q10 supplementation reduces oxidative stress and increases antioxidant enzyme activity in patients with coronary artery disease. Nutrition 2012; 28:250-5.

46. Ognjanovic BI, Markovic SD, Pavlovic SZ, Zikic RV, Stajn AS, Saicic ZS. Combined effects of coenzyme $Q(10)$ and vitamin $E$ in cadmium induced alterations of antioxidant defense system in the rat heart. Environ Toxicol Pharmacol 2006;22:219-24.

47. Safarinejad MR. Efficacy of coenzyme Q10 on semen parameters, sperm function and reproductive hormones in infertile men. J Urol 2009;182:237-48.

48. Safarinejad MR, Safarinejad S, Shafiei N, Safarinejad S. Effects of the reduced form of coenzyme Q10 (ubiquinol) on semen parameters in men with idiopathic infertility: a double-blind, placebo controlled, randomized study. J Urol 2012;188:526-31.

49. Safarinejad MR. The effect of coenzyme Q10 supplementation on partner pregnancy rate in infertile men with idiopathic oligoasthenoteratozoospermia: an open-label prospective study. Int Urol Nephrol 2012;44:689-700.

50. Nadjarzadeh A, Shidfar F, Amirjannati N, Vafa MR, Motevalian SA, Gohari MR, et al. Effect of Coenzyme Q10 supplementation on antioxidant enzymes activity and oxidative stress of seminal plasma: a double-blind randomised clinical trial. Andrologia 2014;46:177-83.

51. Nadjarzadeh A, Sadeghi MR, Amirjannati N, Vafa MR, Motevalian SA, Gohari MR, et al. Coenzyme Q10 improves seminal oxidative defense but does not affect on semen parameters in idiopathic oligoasthenoteratozoospermia: a randomized double-blind, placebo controlled trial. J Endocrinol Invest 2011;34:e224-8.

52. Thakur AS, Littarru GP, Funahashi I, Painkara US, Dange NS, Chauhan P. Effect of ubiquinol therapy on sperm parameters and serum testosterone levels in oligoasthenozoospermic infertile men. J Clin Diagn Res 2015;9:BC01-3.

53. Lafuente R, Gonzalez-Comadran M, Sola I, Lopez G, Brassesco M, Carreras R, et al. Coenzyme Q10 and male infertility: a metaanalysis. J Assist Reprod Genet 2013;30:1147-56.

54. Olayemi FO. A review on some causes of male infertility. Afr J Biotechnol 2010;9:2834-42.

55. Khan MS, Zaman S, Sajjad M, Shoaib M, Gilani G. Assessment of the level of trace element zinc in seminal plasma of males and evaluation of its role in male infertility. Int J Appl Basic Med Res
2011;1:93-6.

56. Favier AE. The role of zinc in reproduction: hormonal mechanisms. Biol Trace Elem Res 1992;32:363-82.

57. Freedman LP. Anatomy of the steroid receptor zinc finger region. Endocr Rev 1992;13:129-45.

58. Kothari RP, Chaudhari AR. Zinc levels in seminal fluid in infertile males and its relation with serum free testosterone. J Clin Diagn Res 2016;10:CC05-8.

59. Hadwan MH, Almashhedy LA, Alsalman AR. Study of the effects of oral zinc supplementation on peroxynitrite levels, arginase activity and NO synthase activity in seminal plasma of Iraqi asthenospermic patients. Reprod Biol Endocrinol 2014;12:1.

60. Hadwan MH, Almashhedy LA, Alsalman AR. Oral zinc supplementation restores high molecular weight seminal zinc binding protein to normal value in Iraqi infertile men. BMC Urol 2012;12:32.

61. Raigani M, Yaghmaei B, Amirjannti N, Lakpour N, Akhondi MM, Zeraati $\mathrm{H}$, et al. The micronutrient supplements, zinc sulphate and folic acid, did not ameliorate sperm functional parameters in oligoasthenoteratozoospermic men. Andrologia 2014;46:95662.

62. Flohe L. Selenium in mammalian spermiogenesis. Biol Chem 2007;388:987-95.

63. Safarinejad MR, Safarinejad S. Efficacy of selenium and/or Nacetyl-cysteine for improving semen parameters in infertile men: a double-blind, placebo controlled, randomized study. J Urol 2009;181:741-51.

64. Keskes-Ammar L, Feki-Chakroun N, Rebai T, Sahnoun Z, Ghozzi H, Hammami S, et al. Sperm oxidative stress and the effect of an oral vitamin $\mathrm{E}$ and selenium supplement on semen quality in infertile men. Arch Androl 2003;49:83-94.

65. Ibrahim HA, Zhu Y, Wu C, Lu C, Ezekwe MO, Liao SF, et al. Selenium-enriched probiotics improves murine male fertility compromised by high fat diet. Biol Trace Elem Res 2012;147:251-60.

66. Ghasemzadeh A, Karkon-Shayan F, Yousefzadeh S, Naghavi-Behzad M, Hamdi K. Study of pentoxifylline effects on motility and viability of spermatozoa from infertile asthenozoospermic males. Niger Med J 2016;57:324-8.

67. Amer M, Metawae B, Hosny H, Raef A. Beneficial effect of adding pentoxifylline to processed semen samples on ICSI outcome in infertile males with mild and moderate asthenozoospermia: a randomized controlled prospective crossover study. Iran J Reprod Med 2013;11:939-44.

68. Nabi A, Khalili MA, Fesahat F, Talebi A, Ghasemi-Esmailabad S. Pentoxifylline increase sperm motility in devitrified spermatozoa from asthenozoospermic patient without damage chromatin and DNA integrity. Cryobiology 2017;76:59-64.

69. Safarinejad MR. Effect of pentoxifylline on semen parameters, 
reproductive hormones, and seminal plasma antioxidant capacity in men with idiopathic infertility: a randomized double-blind placebo-controlled study. Int Urol Nephrol 2011;43:315-28.

70. Oliva A, Dotta A, Multigner L. Pentoxifylline and antioxidants improve sperm quality in male patients with varicocele. Fertil Steril 2009;91(4 Suppl):1536-9.

71. Wirleitner B, Vanderzwalmen P, Stecher A, Spitzer D, Schuff M, Schwerda $D$, et al. Dietary supplementation of antioxidants improves semen quality of IVF patients in terms of motility, sperm count, and nuclear vacuolization. Int J Vitam Nutr Res 2012;82:3918.

72. Alahmar AT. Effect of vitamin C, vitamin E, zinc, selenium, and coenzyme Q10 in infertile men with idiopathic oligoasthenozoospermia. Int J Infertil Fetal Med 2017;8:45-9.

73. Abad C, Amengual MJ, Gosalvez J, Coward K, Hannaoui N, Benet $J$, et al. Effects of oral antioxidant treatment upon the dynamics of human sperm DNA fragmentation and subpopulations of sperm with highly degraded DNA. Andrologia 2013;45:211-6.

74. Piomboni P, Gambera L, Serafini F, Campanella G, Morgante G, De Leo V. Sperm quality improvement after natural anti-oxidant treatment of asthenoteratospermic men with leukocytospermia. Asian J Androl 2008;10:201-6.

75. Gopinath P, Kalra B, Saxena A, Malik S, Kochhar K, Kalra S, et al. Fixed dose combination therapy of antioxidants in treatment of idiopathic oligoasthenozoospermia: results of a randomized, double-blind, placebo-controlled clinical trial. Int J Infertil Fetal Med 2013;4:6-13.

76. Tremellen K, Miari G, Froiland D, Thompson J. A randomised control trial examining the effect of an antioxidant (Menevit) on pregnancy outcome during IVF-ICSI treatment. Aust N Z J Obstet Gynaecol 2007;47:216-21.

77. Busetto GM, Koverech A, Messano M, Antonini G, De Berardinis E, Gentile V. Prospective open-label study on the efficacy and tolerability of a combination of nutritional supplements in primary infertile patients with idiopathic astenoteratozoospermia. Arch Ital Urol Androl 2012;84:137-40.

78. Ghanem H, Shaeer O, El-Segini A. Combination clomiphene citrate and antioxidant therapy for idiopathic male infertility: a randomized controlled trial. Fertil Steril 2010;93:2232-5.

79. Comhaire FH, Christophe AB, Zalata AA, Dhooge WS, Mahmoud AM, Depuydt CE. The effects of combined conventional treatment, oral antioxidants and essential fatty acids on sperm biology in subfertile men. Prostaglandins Leukot Essent Fatty Acids 2000;63:159-65.

80. Akpinar E, Bashan I, Bozdemir N, Saatci E. Which is the best anthropometric technique to identify obesity: body mass index, waist circumference or waist-hip ratio? Coll Antropol 2007;31: 387-93.

81. Mayorga-Torres BJM, Camargo M, Cadavid AP, du Plessis SS, Cardona Maya WD. Are oxidative stress markers associated with unexplained male infertility? Andrologia 2017;49:e12659.

82. Hosen MB, Islam MR, Begum F, Kabir Y, Howlader MZ. Oxidative stress induced sperm DNA damage, a possible reason for male infertility. Iran J Reprod Med 2015;13:525-32.

83. Tremellen K. Oxidative stress and male infertility: a clinical perspective. Hum Reprod Update 2008;14:243-58.

84. Crha I, Hruba D, Ventruba P, Fiala J, Totusek J, Visnova H. Ascorbic acid and infertility treatment. Cent Eur J Public Health 2003;11:637.

85. Sheweita SA, Tilmisany AM, Al-Sawaf H. Mechanisms of male infertility: role of antioxidants. Curr Drug Metab 2005;6:495-501.

86. Jacob RA. The integrated antioxidant system. Nutr Res 1995;15:75566.

87. Singh A, Jahan N, Radhakrishnan G, Banerjee BD. To evaluate the efficacy of combination antioxidant therapy on oxidative stress parameters in seminal plasma in the male infertility. J Clin Diagn Res 2016;10:QC14-7.

88. Safarinejad MR, Shafiei N, Safarinejad S. A prospective doubleblind randomized placebo-controlled study of the effect of saffron (Crocus sativus Linn.) on semen parameters and seminal plasma antioxidant capacity in infertile men with idiopathic oligoasthenoteratozoospermia. Phytother Res 2011;25:508-16.

89. Janevic T, Kahn LG, Landsbergis P, Cirillo PM, Cohn BA, Liu X, et al. Effects of work and life stress on semen quality. Fertil Steril 2014; 102:530-8. 